\title{
Estudo da lixiviação do carbofurano em diferentes perfis de coluna de latossolo vermelho
}

\author{
Camila Faria Silva, Jordana Teodoro Bernardes, Adilson Correia Goulart*, Simone Machado \\ Goulart
}

Instituto Federal de Goiás, Itumbiara, 75524-010, Goiás, Brasil. *adilson.goulart@ifg.edu.br

Recebido: 14 julho 2018 / Aceito: 9 setembro 2018 / Publicado online: 12 setembro 2018

\begin{abstract}
Resumo
Esta pesquisa objetivou avaliar a lixiviação do carbofurano ao longo do perfil de colunas de latossolo vermelho. Para o estudo foram utilizadas 4 colunas preenchidas com solo sendo aplicada $150 \mathrm{mg} \mathrm{L}^{-1}$ do produto comercial Furadan ${ }^{\circledR}$. Foram avaliados a simulação de três níveis de precipitação $(20,60,100 \mathrm{~mm})$ de chuva e três faixas de profundidade $(0-5,25-30,45-50 \mathrm{~cm})$ por um período de 24h. A extração do Furadan ${ }^{\circledR}$ foi realizada por meio da Extração Sólido-Líquido com Partição a Baixa Temperatura (ESL-PBT), sendo determinado por Cromatografia Líquida de Alta Eficiência (CLAE-UV). Na simulação de volumes 20 e 60 $\mathrm{mm}$ de chuva, a lixiviação do Furadan ${ }^{\circledR}$ aconteceu somente em camadas superficiais do solo $(0-5 \mathrm{~cm})$. Entretanto, à $100 \mathrm{~mm}$ de chuva, o produto foi lixiviado até a camada intermediária do solo $(25-30 \mathrm{~cm})$. Desta forma, verificou-se que a capacidade de lixiviação do Furadan ${ }^{\circledR}$ foi maior ao simular $100 \mathrm{~mm}$ de precipitação. Novos estudos, com outros tipos de solo e maior observação temporal, são recomendados para o potencial de incorporação desse agrotóxico no perfil do solo.
\end{abstract}

Palavras-chave: agrotóxico, solo, contaminação ambiental, ESL-PBT, CLAE-UV.

\section{Study of leaching of carbofuran in different profiles of red latosol column}

\begin{abstract}
This research aimed to evaluate the leaching of carbofuran along the profile of red latosol columns. For the study, 4 columns filled with soil were used and $150 \mathrm{mg} \mathrm{L}^{-1}$ of the commercial product Furadan ${ }^{\circledR}$ was applied. The simulation of three levels of precipitation $(20,60,100 \mathrm{~mm})$ of rainfall and three depth ranges $(0-5,25-30,45-50 \mathrm{~cm})$ for a period of $24 \mathrm{~h}$ was evaluated. The extraction of Furadan ${ }^{\circledR}$ was carried out by means of the Solid-Liquid Extraction with Low Temperature Partitioning (SLE-LTP), being determined by High Performance Liquid Chromatography (HPLC-UV). In the simulation of 20 and $60 \mathrm{~mm}$ rain volumes, Furadan ${ }^{\circledR}$ leaching occurred only in superficial soil layers $(0-5 \mathrm{~cm})$. However, at $100 \mathrm{~mm}$ of rain, the product was leached to the middle layer of the soil $(25-30 \mathrm{~cm})$. In this way, it was verified that the leaching capacity of Furadan ${ }^{\circledR}$ was greater when simulating $100 \mathrm{~mm}$ of precipitation. New studies, with other types of soil and greater temporal observation, are recommended for the potential of incorporation of this pesticide into the soil profile.
\end{abstract}

Keywords: agrotoxic, soil, environmental contamination, SLE-LTP, HPLC-UV.

\section{Introdução}

Na aplicação dos agrotóxicos, além de atingir o seu alvo, grande parte do princípio ativo alcança outras regiões que não são alvos, como, por exemplo, os componentes ambientais ar, solo, recursos hídricos e mesmo que sejam em concentrações baixas estas substâncias poderão ocasionar malefício aos ecossistemas podendo gerar impactos negativos ao meio ambiente (Rebelo e Caldas, 2014; Osorio et al., 2017). De todos os componentes ambientais o solo é o mais atingido pelas moléculas de agrotóxico uma vez contaminado funciona como via de contaminação de águas superficiais, por meio do escoamento superficial, e também de águas subterrâneas, via processo de lixiviação (Oliveira e Brighenti, 2011). Em decorrência disso, vários estudos têm demonstrado contaminação generalizada em diferentes ambientes, tais como, solo, sedimento, recursos hídricos (Gomes e Barizon, 2014) e até mesmo em fluidos humanos, como, por exemplo, sangue e urina (Carneiro et al., 2015).

O princípio ativo carbofurano pertence a classe química dos carbamatos e possui nomenclatura química oficial(2,3-dihidro2,2-dimetilbenzofurano-7-metilcarbamato). É utilizado para o controle de insetos, cupins e nematóides podendo ser aplicado nas culturas de algodão, arroz, feijão, milho, trigo, tomate, repolho, cana-de-açúcar, banana, batata, café, cenoura e fumo (ANVISA, 2018). É uma substância considerada muito perigosa ao meio ambiente e extremamente tóxica aos seres vivos. A maneira de aplicação do composto pode ser incorporando ao solo ou no trato de sementes (IUPAC, 2018). Estudos teóricos aplicando modelos matemáticos $\mathrm{e}$ as propriedades físico-químicas do carbofurano, apontam que o 
princípio ativo possui alto potencial de poluição das águas superficiais e subterrâneas (Cabrera et al., 2008; Rebelo e Caldas 2014; Bedmar et al., 2015) demonstrando a necessidade de estudos práticos em relação a dinâmica deste agrotóxico no solo.

O solo selecionado para verificar o potencial de lixiviação do carbofurano é do tipo latossolo vermelho. Este tipo de solo é um dos mais escolhidos para pesquisas na agricultura brasileira, ocupando $32 \%$ de todo território nacional. Suas caraterísticas físicas facilitam o processo de implementação agrícola o que potencializa sua utilização para o cultivo de diferentes tipos de cultura (Souza e Lobato, 2017).

Essa pesquisa objetivou investigar a lixiviação do carbofurano ao longo do perfil de colunas preenchidas com latossolo vermelho.

\section{Material e Métodos}

\section{Preparação e preenchimento das colunas de solo}

As colunas foram confeccionadas de acordo com Faria (2013). Utilizando colunas de PVC de 10x60 cm. Antes do preenchimento com amostras de latossolo vermelho deformadas, as colunas foram parafinadas internamente para que não se formassem rotas preferenciais no deslocamento da água. A parcela do solo foi coletada em local onde não existia histórico de aplicação de agrotóxicos (Coordenadas: Latitude 18²6'10"S e Longitude 49 $12^{\prime} 43^{\prime \prime} 43 \mathrm{~W}$ ). As colunas foram preenchidas com o solo peneirado (peneira aço inox malha 2 $\mathrm{mm}$ ) até $\pm 50 \mathrm{~cm}$. Com o intuito de reproduzir uma parcela fiel do perfil do solo as colunas foram saturadas com água destilada e posteriormente drenadas.

\section{Aplicação do Furadan ${ }^{\circledR}$, precipitação e coleta das amostras}

O Furadan ${ }^{\circledR}$ foi adquirido em loja de produtos agrícola especificamente para fins de pesquisa. Uma solução na concentração de $150 \mathrm{mg} \mathrm{L}^{-1}$ foi preparada com o produto e posteriormente aplicada conforme orientação do fabricante. Foram utilizadas 4 colunas, em uma destas não se aplicou dose do produto Furadan ${ }^{\circledR}$ sendo identificada como branco.

Foi investigada a simulação de três volumes de precipitação (20, 60 e $100 \mathrm{~mm}$ ), individualmente, utilizando um frasco simples em polipropileno graduado de boca larga com tampa e com furos de pequeno diâmetro na tampa.

O potencial de lixiviação do Furadan ${ }^{\circledR}$ foi determinado em três perfis $(0-5,25-30,45-50 \mathrm{~cm})$. As amostras de solo foram coletadas após $24 \mathrm{~h}$ e, deixada em repouso a temperatura ambiente até estarem completamente secas e submetidas à ESL-PBT.

\section{ESL-PBT e análise cromatográfica}

Na extração e quantificação do carbofurano foi utilizada a metodologia otimizada por Goulart (2017). Desta forma, 1,0000 grama do solo seco foi pesado em frasco de vidro com capacidade de $22 \mathrm{~mL}$. Em seguida adicionou 1,5 mL de água deionizada e 4,0 mL de acetonitrila (ACN) agitando o sistema por 30s em agitador de tubos do tipo vortex. Após a agitação as amostras foram deixadas em freezer por um período superior a $2 \mathrm{~h}$ em temperatura de aproximadamente $-20{ }^{\circ} \mathrm{C}$. Posterior ao período de partição retirou-se uma alíquota de 1,0 $\mathrm{mL}$ do sobrenadante para análise cromatográfica. $\mathrm{O}$ equipamento utilizado nas análises tem as seguintes especificações: HPLC alliance Waters e2695 separations module.2998 PDA Detector, gerenciado pelo software Empawer 3. As condições cromatográficas utilizadas foram: Detector de UV a $195 \mathrm{~nm}$; Coluna Kinetex $5 \mu \mathrm{m}$ EVO C 18 150 X 4,6 mm; fase móvel (modo isocrático); Acetonitrila:Água deionizada 35:65 v/v; Vazão da fase móvel: 0,8 $\mathrm{mL} \mathrm{min}^{-1}$; Temperatura da coluna: $35{ }^{\circ} \mathrm{C}$; Volume de injeção: $20 \mu \mathrm{L}$; e Tempo de análise: 7 minutos. O carbofurano foi identificado pelo tempo de retenção do padrão $(5,5 \mathrm{mim})$ no cromatograma.

\section{Preparo da curva analítica}

A quantificação do carbofurano foi obtida pelo método da padronização externa sendo construída uma curva analítica em ACN nas concentrações 5, 10, 30, 50, 70, 100, 150, 170, 200 $m g L^{-1}$, obtendo a função linear $(y=204658 x+716049)$ para calcular as concentrações do princípio ativo.

\section{Resultados e Discussão}

No cromatograma da amostra de solo sem adição de carbofurano (branco), não se verificou a presença desse composto, comprovando a inexistência de interferentes ou contaminantes na matriz no tempo de retenção para este composto. A Figura 1 mostra 3 cromatogramas que representam as análises realizadas nos perfis analisados $0-5$, 25-30, 45-50 cm.
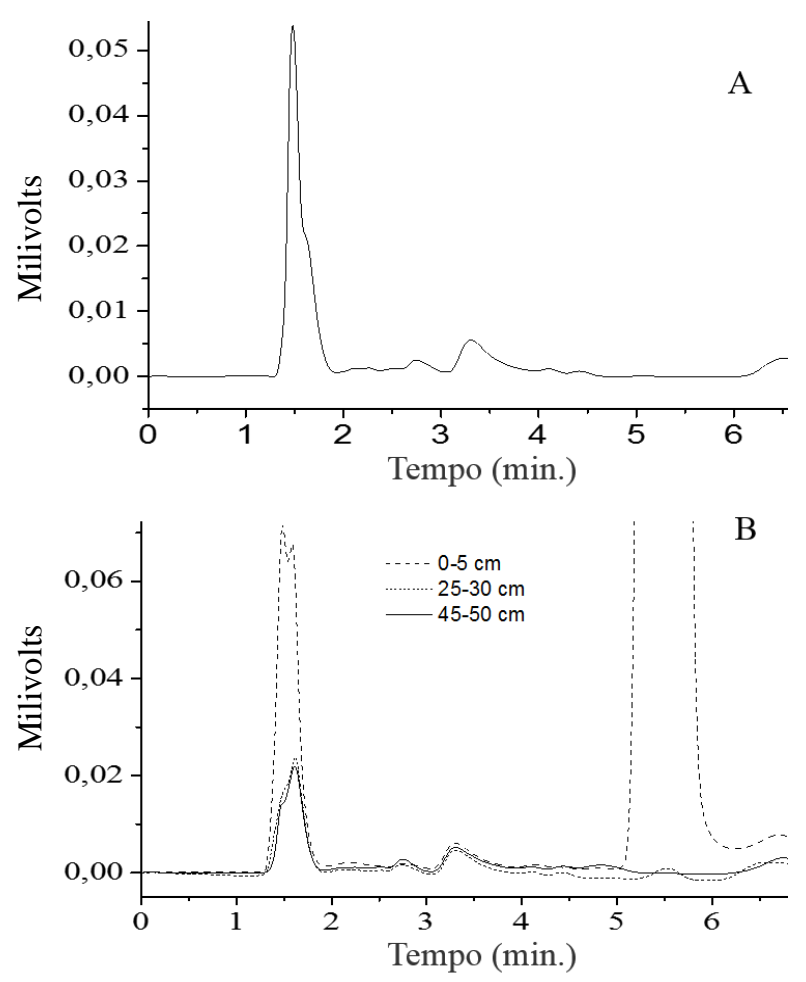

Figura 1. Cromatogramas das amostras de solo coletado das colunas sem adição de carbofurano - branco (A) e com adição (B), nos três perfis das colunas. 
Os resultados cromatográficos, para os três volumes de precipitação, revelaram concentrações superiores a $120 \mathrm{mg} \mathrm{L}$ ${ }^{1}$ no perfil inicial, profundidade $0-5 \mathrm{~cm}$, em todas as colunas analisadas, com exceção do branco. A movimentação das moléculas de carbofurano para o perfil intermediário, profundidade $25-30 \mathrm{~cm}$, não foi verificada ao simular 20 e 60 $\mathrm{mm}$ de precipitação. Esta migração ocorreu somente ao simular o volume de $100 \mathrm{~mm}$. No perfil intermediário desta coluna as análises cromatográficas revelaram concentrações menores que $10 \mathrm{mg} \mathrm{L}^{-1}$. À vista disso, pode se dizer que a capacidade de lixiviação do carbofurano foi maior quando simulou $100 \mathrm{~mm}$ de precipitação.

Segundo Oliveira e Brighenti (2011) o comportamento de um agrotóxico no ambiente está ligado diretamente com suas propriedades físico-química. Ainda segundo os autores o coeficiente de adsorção a matéria orgânica $\left(\mathrm{K}_{\mathrm{oc}}\right)$, o coeficiente de partição octanol-água $\left(\mathrm{K}_{\mathrm{ow}}\right)$ e a solubilidade em água são propriedades que podem influencias no processo de lixiviação dos agrotóxicos. De acordo com a União Internacional de Química Pura e Aplicada (IUPAC, 2018) e Cabrera et al, (2008) o carbofurano apresenta valores de $\mathrm{K}_{\mathrm{oc}}=$ $22 \mathrm{~mL} \mathrm{~g}^{-1}, \mathrm{~K}_{\mathrm{ow}}=1,52$ e solubilidade em água de $351 \mathrm{mg} \mathrm{L}^{-1}$. Desta forma, uma possível explicação para a movimentação do carbofurano, observada nesta pesquisa, pode estar nas propriedades físico-químicas do carbofurano. Isso porque o baixo coeficiente de adsorção a matéria orgânica $\left(\mathrm{K}_{\mathrm{oc}}=22 \mathrm{~mL}\right.$ $\mathrm{g}^{-1}$ ) dificulta sua adsorção a matéria orgânica no solo deixando o princípio ativo livre no ambiente, favorecendo sua movimentação entre os poros do solo; Já o coeficiente de partição octanol-água $\left(\mathrm{K}_{\mathrm{ow}}=1,52\right)$ confere ao princípio ativo carbofurano a característica de molécula hidrofílica, ou seja, maior afinidade com a água e pouca afinidade com os componentes presentes no solo contribuindo para sua mobilidade; $\mathrm{E}$ a alta solubilidade em água $\left(351 \mathrm{mg} \mathrm{L}^{-1}\right) \mathrm{faz}$ com que o carbofurano se ligue mais facilmente as moléculas de água favorecendo a movimentação deste princípio ativo.

Possivelmente devido ao tempo de coleta estabelecido, posterior a 24 horas da aplicação e precipitação, em nenhuma das amostras analisadas foi verificada concentração de carbofurano no último perfil da coluna, profundidade 45-50 $\mathrm{cm}$.

Pesquisas com foco na lixiviação de agrotóxicos tiveram resultados semelhantes aos desta pesquisa. Spadotto et al. (2001), por exemplo, avaliou a lixiviação de 19 agrotóxicos pelo fator de atenuação em latossolo vermelho da região de Guaíra Estado de São Paulo. O estudo levou em consideração as propriedades físico-químicas dos agrotóxicos e do latossolo. Os autores concluíram que o carbofurano foi o segundo agrotóxico de maior lixiviação nos primeiros $60 \mathrm{~cm}$ do solo. A justificativa para esta observação, segundo os autores, está na alta solubilidade em água e na característica lipofílica da molécula de carbofurano.

Já o estudo de Kazemi et al. (2009) avaliaram a campo a movimentação vertical do carbofurano em solos do tipo Menfro limo-argiloso, localizados em New Franklin, Estado americano de Missouri. Os autores constataram que a lixiviação do carbofurano dependia da quantidade e atraso da simulação de precipitação. Observaram também que após a aplicação a concentração do princípio ativo era maior na parte superficial do solo. Após alguns dias da aplicação foram encontradas concentrações de carbofurano em perfis mais profundos do solo de $35 \mathrm{a} 45 \mathrm{~cm}$.

Com o objetivo de entender os mecanismos de transporte para o desenvolvimento de um modelo matemático capaz de reproduzir a destinação do carbofurano no solo, Hmimou et al. (2014) realizaram estudo semelhante aplicando doses do produto comercial Furadan ${ }^{\circledR}$ e em seguida simulações de precipitação em parcelas de solo de $4 \times 5 \mathrm{~m}^{2}$. Em seus resultados os autores afirmam que após 25 dias de lixiviação o carbofurano foi distribuído em praticamente toda a matriz do solo. Ressaltam também que maiores concentrações do composto foram encontradas na camada superficial de $0-20$ $\mathrm{cm}$, no entanto foram encontrados resíduos da substância em níveis mais profundos de $45-60 \mathrm{~cm}$.

\section{Conclusões}

Nos padrões estabelecidos e metodologia adotada, verificou-se a lixiviação do carbofurano no perfil do solo em função da quantidade de precipitação. Além disso, é possível inferir que mesmo utilizando baixos volumes de precipitação o princípio ativo carbofurano pode lixiviar em camadas superficiais do solo. $\mathrm{O}$ estudo demonstrou também que ao simular $100 \mathrm{~mm}$ de precipitação a lixiviação do carbofurano foi favorecida. Novos estudos, adotando um maior período para a realização das coletas e maiores volumes de precipitação, devem ser realizados para melhor entendimento da dinâmica deste princípio ativo em latossolo vermelho.

\section{Agradecimentos}

Os autores agradecem ao Laboratório Multiusuário do Pontal da Universidade Federal de Uberlândia por fornecer o equipamento e suporte técnico para os experimentos envolvendo a Cromatografia Líquida de Alta Eficiência.

\section{Referências}

ANVISA. 2018. Agência Nacional de Vigilância Sanitária; RE nº 899/2003: Guia para validação de métodos analíticos e bioanalíticos, Ministério da Saúde: Brasil, 2003. Disponível em: $<$ http://portal.anvisa.gov.br/registros-e-

autorizacoes/agrotoxicos/produtos/monografia-de-agrotoxicos>. Acesso em: 23 abr. 2018.

ANVISA. 2018. Agência Nacional de Vigilância Sanitária. Monografias de

agrotóxicos. Disponível em: <http://portal.anvisa.gov.br/registros-eautorizacoes/agrotoxicos/produtos/monografia-de-agrotoxicos $>$. Acesso em: 28 ago. 2018.

Bedmar, F.; Gianelli, V.; Angelini, H.; Viglianchino, L. 2015. Riesgo de contaminación del agua subterránea con plaguicidas en la cuenca del arroyo El Cardalito, Argentina. Revista de investigaciones agropecuarias, 41(1): 70-82.

Cabrera, L.C.; Costa, F.P.; Primel, E.G. 2008. Estimativa de risco de contaminação das águas por pesticidas na região sul do estado do RS. Química Nova, 31(8): 1982-1986.

Carneiro, F. F.; Augusto, L.G.S.; Rigotto, R. M.; Friedrich, K.; Búrigo, A.C. 2015. Dossiê ABRASCO: um alerta sobre os impactos dos agrotóxicos na saúde. Rio de Janeiro: EPSJV; São Paulo: Expressão Popular, 98p. Disponível em: <https://www.arca.fiocruz.br/handle/icict/26221>. Acesso em: 07 mai. 2018.

Faria, A.T. 2013. Sorção, dessorção, meia-vida e lixiviação do tebuthiuron em latossolos brasileiros. Dissertação Mestrado, Universidade Federal de Viçosa, Viçosa. 71p.

Gomes, M.A.; Barizon, R.R.M. 2014. Panorama da contaminação ambiental por agrotóxicos e nitrato de origem agrícola no Brasil: cenário 1992/2011. 
Embrapa Meio Ambiente: São Paulo, Embrapa Meio Ambiente. Disponível em: <https://www.embrapa.br/busca-de-publicacoes//publicacao/987245/panorama-da-contaminacao-ambiental-por-

agrotoxicos-e-nitrato-de-origem-agricola-no-brasil-cenario-19922011> Acesso em: 18 de mai. 2018.

Goulart, A.C. Goulart, S.M.; Medeiros, M.S.; Santos, J.P.V. 2018. Avaliação da contaminação ambiental por carbofurano em solo proveniente do cultivo de cana-de-açúcar. Tecno-lógica, Santa Cruz do Sul, 22(2): 187193.

Hmimou, A.; Maslouhi, A.; Tamoh, K.; Candela, L. 2014. Experimental monitoring and numerical study of pesticide (carbofuran) transfer in an agricultural soil. Comptes Rendus Geoscience, 346(9-10): 255-261.

IUPAC, 2018. International Union of Pure and Applied Chemistry. Footprint pesticides properties database. Disponível em: 〈https://sitem.herts.ac.uk/aeru/ppdb/en/Reports/118.htm〉. Acesso em 10 jun. 2018.

Kazemi, H.V.; Anderson, S.H.; Goyne, K.W.; Gantzer, C.J. 2009. Aldicarb and carbofuran transport in a Hapludalf influenced by differential antecedent soil water content and irrigation delay. Chemosphere, 74(2): 265-273.

Oliveira, M.F.; Brighenti, A.M. 2011. Comportamento dos herbicidas no ambiente In: Oliveira J.R.; Constantin, J.; Inoue, M.H. (Ed.). Biologia e Manejo de Plantas Daninhas. Curitiba, Omnipax, 263-304p.

Osorio, J.L.S.;Zamora, J.V.M.; Álvarez, N.R.; Bidleman, T.F. 2017. Organochlorine pesticides in residential soils and sediments within two main agricultural areas of northwest Mexico: Concentrations, enantiomer compositions and potential sources. Chemosphere, 173(Abr.), 275-287.

Rebelo, R.M.; Caldas, E.D. 2014. Avaliação de risco ambiental de ambientes aquáticos afetados pelo uso de agrotóxicos. Química Nova, 37(7): 11991208.

Santos, J.L.O.; Leite, O.D. 2016. Avaliação do risco de contaminação de águas subterrâneas na região oeste da Bahia pelo inseticida carbofuran, empregando os modelos de Attenuation Factor (AF) e Retardation Factor (RF). Orbital: Electron. Journal Chemistry, 8(1): 28-35.

Souza, D.M.G.; Lobato, E. 2018. Latossolos. Brasília, EMBRAPA. Disponível

em:<http://www.agencia.cnptia.embrapa.br/Agencia16/AG01/arvore/A G01_96_10112005101956.html>. Acesso em: 14 de mai. 2018.

Spadotto, C.; Filizola, H.; Gomes. 2001. Avaliação do potencial de lixiviação de pesticidas em latossolo da região de Guaíra, SP. Pesticidas: Revista de Ecotoxicologia. e Meio Ambiente, 11(2001): 127-136. 